\title{
Study of Lymphatic Metastatic Patterns in Gastric Carcinoma According to Tumor Location
}

\author{
JITENDRA TIWARI, ISSEI KODAMA, JINRYO TAKEDA \\ AND KAZUO SHIROUZU \\ Department of Surgery, Kurume University School of Medicine, \\ Kurume 830-0011, Japan
}

\begin{abstract}
Summary: A total of 226 cases of advanced gastric cancer which occupied only one third of the stomach were analyzed in order to clarify whether and how lymphatic spread differed according to the tumor location and gross type of tumor. Out of the 226 patients, 45 cases had tumor in the upper third, 74 cases had it in the middle third, and 107 cases had it in the lower third of the stomach. The incidence of lymph node metastasis was found to be much higher for the tumors located in the lesser curvature (51.6\%), greater curvature and posterior wall $(54.4 \%)$, as compared to the tumors located in the anterior wall $(28.0 \%)$. The tumors located in the upper third of the stomach did not show any metastasis in the $\mathrm{N}_{3}$ node, while the tumors located in the lower third of the stomach did not show any metastasis in the left cardial nodes, short gastric nodes, and the nodes along the left gastroepiploic vessels. Similarly, the tumors from the middle third of the stomach did not invade the left cardial nodes.
\end{abstract}

Key words gastric cancer, lymph node, metastasis

\section{INTRODUCTION}

The prognosis of surgically treated gastric carcinoma has been poor in most parts of the world except Japan, and the key to the success of Japanese surgeons has been their early diagnosis and wide excision with extensive lymphadenectomy [1-4].

This study was conducted in our department to ascertain the lymph node metastatic pathways of gastric carcinoma according to tumor location. Since the incidence of lymphatic metastasis is very low in mucosal and submucosal gastric cancers, they were excluded from the study.

\section{MATERIALS AND METHODS}

A total number of 226 cases of advanced gastric carcinoma, which were treated in the surgical department of Kurume University Medical School from 1980 to 1991, were selected for this study. For a better understanding of nodal metastasis, the Japanese classification of the stomach [5] was used, according to which any tumor which has penetrated the muscularis propria is an advanced gastric cancer, because the possibility of lymph node metastasis becomes three times higher in these cases.

The Japanese classification of the stomach into (C) upper third, (M) middle third, and (A) lower third, was used. The tumor locations were further divided into the anterior wall, posterior wall, lesser and greater curvature for each of these subgroups. The pattern of lymphatic metastasis was studied in relation to the tumor location, size, gross type, depth, histological type, and lymphatic invasion, also based on the Japanese classification of the stomach [5]. The majority of these patients underwent a curative resection $(86.3 \%)$ which means that no evidence of tumor was left behind macroscopically or microscopically. Depending upon the tumor location, histological type, intra-operative staging and the estimated operative risk of the individual patient, a total or partial distal gastrectomy was performed. The omen- 
tum was removed in all the cases, while splenectomy was performed in most of the cases of total gastrectomy.

While the patient was still in the operating room the stomach was opened either along the greater or lesser curvature to ascertain the exact tumor location, the size, gross type, and the distance from the resection margin. All the lymph nodes were picked up by the surgical staff. The lymph nodes were categorized according to the lymph node stations in which they were found, and then the specimen was handed over to the pathologist. Paraffin sections of each lymph node were stained to determine the metastasis and its preferred pathway. All the resected and metastatic lymph nodes were counted.

\section{RESULTS}

A total of 1,481 gastrectomies were performed in the Department of Surgery, Kurume University Medical School, from 1980 to 1991, out of which 226 cases were found suitable for this study. The age ranged from 26 years to 86 years, with a mean age of 66.6 years. There were 143 male and 83 female patients.

Out of all the patients, 107 cases $(48.4 \%)$ had a tumor in the lower third, 74 cases $(32.7 \%)$ had it in the middle third, and 45 cases $(19.9 \%)$ had it in the upper third of the stomach.

The incidence of lymph node metastasis was found to be much higher for the tumors located in the lesser curvature $(51.6 \%)$, greater curvature $(45.6 \%)$

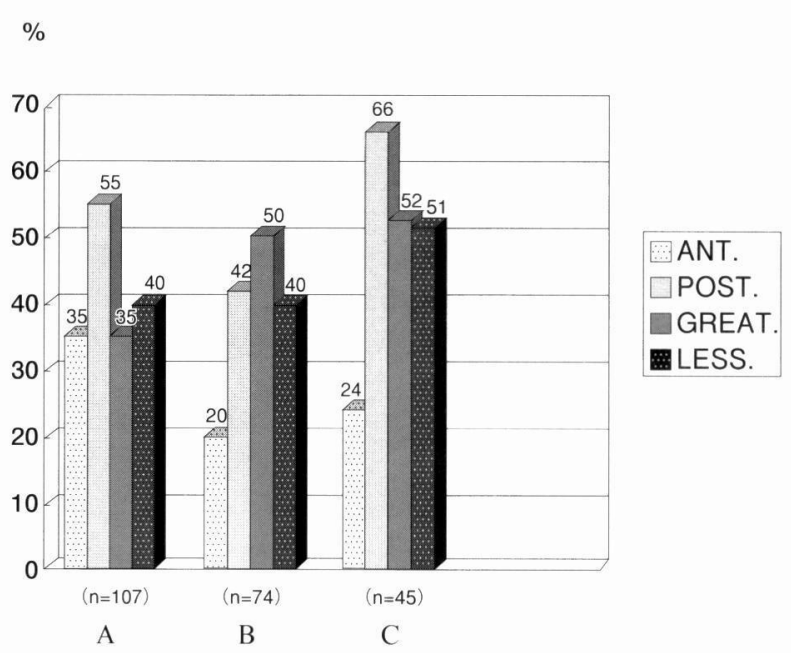

Fig. 1. Lymph node metastasis in relation to tumor locations.

A: upper third B: middle third C: lower third and posterior wall $(54.4 \%)$, as compared to the tumors located in the anterior wall $(28.0 \%)$ in all the subgroups (Fig. 1).

The tumors located in the upper third of the stomach did not show any metastasis in the $\mathrm{N}_{3}$ node, (lymph nodes of group 12 to 15 ) while the tumors located in the lower third of the stomach did not show any metastasis in the left cardial nodes, short gastric nodes, and the nodes along the left gastroepiploic vessels. The incidence of right cardial node involvement was only $2.1 \%$ for these tumors. Similarly, the tumors from the middle third of the stomach did not invade the left cardial nodes.

The incidence of nodal metastasis was less than $20 \%$ for a proliferative lesion, while it was $53.2 \%$ and $57.1 \%$ for an ulcerative or ulceroinfiltrative lesion.

The incidence of mucinous carcinoma was $3.9 \%$ of the total, but $77.7 \%$ of these tumors showed nodal metastasis, while only $30 \%$ of cases of papillary carcinoma (4.4\% of total) showed nodal metastasis. The incidence of nodal metastasis for well differentiated, moderately differentiated, and poorly differentiated carcinoma was $39.8,59.4$, and $49.6 \%$, respectively.

A tumor of less than $2 \mathrm{~cm}$ in diameter did not show significant node metastasis (less than 9\%), but node metastasis was more than $40 \%$ for the larger tumors. There was no significant relationship between

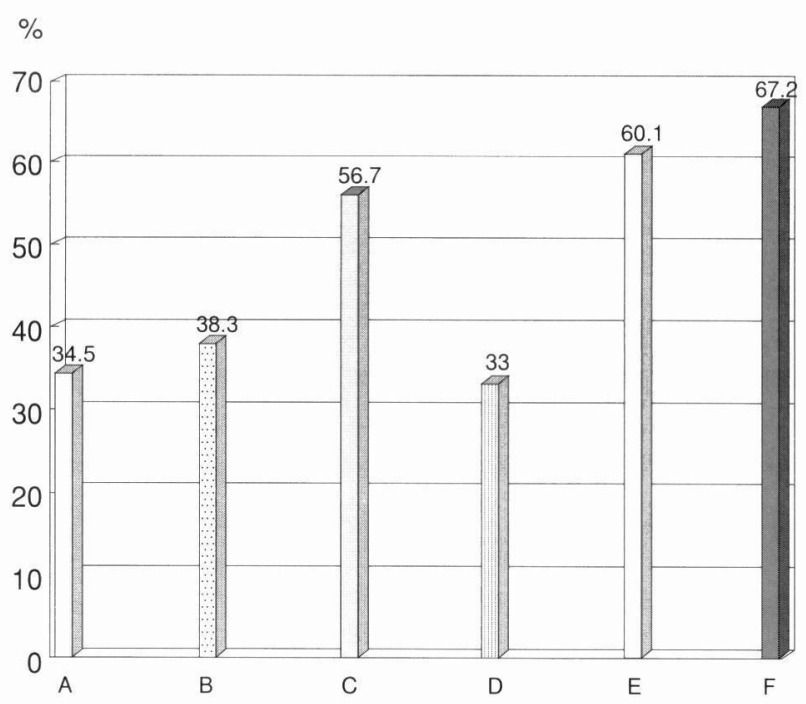

Fig. 2. Lymph node metastasis in relation to tumor depth and lymphatic invasion.

A: muscularis propria B: subserosa C: serosa

D: mild infiltration $\mathrm{E}$ : moderate infiltration

F: severe infiltration 
the incidence of nodal metastasis and tumor size in the cases with tumors greater than $2 \mathrm{~cm}$.

The incidence of nodal involvement was $34.5 \%$ for tumors invading the muscularis propria and $38.3 \%$ for tumors involving the subserosa, but it was higher for the tumors involving the serosa $(56.7 \%)$ (Fig. 2A-C).

The incidence of nodal metastasis was $33.0 \%$ for tumors having mild lymphatic infiltration, while it was much higher for moderate and marked lymphatic infiltration (60.1 and 67.2\%) (Fig. 2D-F).

\section{DISCUSSION}

The present study showed that the incidence of tumor location was highest for the distal third of the stomach $(48.4 \%)$ and that most of the cases underwent curative resection, similar to the findings of many other workers $[2,6,7]$.

The tumors located in the lesser and greater curvature, and the posterior wall showed much higher incidence of nodal metastasis $(51.6,45.6$ and $54.4 \%$, respectively), as compared to the tumors of the anterior wall $(28.0 \%)$. This suggests a need for more aggressive lymphadenectomy for the tumors located in or near the curvatures and the posterior wall of the stomach, while tumors in the anterior wall of the stomach can be treated by a less aggressive approach.

The tumors located in the upper third of the stomach did not show any metastasis in the paraaortic, para-colic, superior mesenteric and retro-pancreatic nodes. A previous study by Jaehne et al. [8] has also shown a very low incidence of gastric carcinoma metastasis in these nodes $(2.6 \%)$. Their study shows that $92.4 \%$ of the metastatic nodes in cases of gastric carcinoma are found in the perigastric region, similar to the results of the present study. Therefore a $\mathrm{D}_{4}$ lymphadenectomy for a small upper third gastric carcinoma becomes an overtreatment, and needs a critical reevaluation in a controlled randomized trial.

The tumors of the middle third of the stomach show no involvement of left cardial nodes, suggesting a near total gastrectomy to be a useful option for these tumors, rather than a total gastrectomy.

The incidence of nodal metastasis was three times higher for an ulcerative tumor $(53.2 \%)$ or infiltrative tumor $(57.1 \%)$, as compared to a proliferative tumor $(20 \%)$. Yamao et al. [9] have shown a similar result for early gastric cancer in their study.

The incidence of metastasis increased with the depth of the tumor, being $34.5 \%$ for tumor invading the muscularis propria, $38.3 \%$ for tumors involving the subserosa and $56.7 \%$ for tumors involving the serosa. Bunt et al. [10] have also reported that the metastatic propensity to lymph nodes increases significantly with increasing depth of the tumor invasion.

The tumors of diameter less than $2 \mathrm{~cm}$ did not show significant nodal metastasis (i.e. less than 9\%), while tumors larger than that had a $40 \%$ incidence of nodal metastasis. This is quite a different finding from other studies and suggests a less aggressive approach for tumors which are less than $2 \mathrm{~cm}$ in diameter, irrespective of their depths. But tumors larger than that need a very aggressive lymphadenectomy due to $40 \%$ incidence of nodal metastasis.

The extent of lymphatic invasion also influenced the nodal metastasis. The incidence of nodal metastasis was $33.0 \%$ for the cases with mild lymphatic infiltration while it was $67.2 \%$ for the cases with severe lymphatic infiltration. Yamao et al. [9] have also shown lymphatic invasion to be an independent risk factor for lymph node metastasis.

\section{CONCLUSION}

From the above study we conclude that tumors of less than $2 \mathrm{~cm}$ in diameter, situated anywhere on the anterior wall of the stomach, can be treated by less aggressive lymphadenectomy, irrespective of the tumor depth, as the chances of nodal metastasis are very low for these tumors. Therefore, the indication for a $\mathrm{D}_{4}$ dissection for a small upper third gastric carcinoma needs a critical re-evaluation. The size, ulceration and lymphatic infiltration remain important risk factors for lymph node metastasis.

\section{REFERENCES}

1. Wanger PK, Ramaswamy A, Ruschoff J, Schmitz Moormann P, and Rothmund M. Lymph node counts in the upper abdomen: anatomical basis for lymphadenectomy in gastric cancer. Br J Surg 1991; 78:825-827.

2. Jatzko GR, Lisborg PH, Denk H, Kilimpfinger M, and Stettner HM. A 10 year experience with Japanese type radical lymph node dissection for gastric cancer outside of Japan. Cancer 1995; 76:1302-1312.

3. Ravichandran D, Lamah M, Carty NJ, and Johnson CD. Extended lymph node dissection $\left(\mathrm{D}_{2}\right.$ resection) should now be performed routinely in the curative surgical treatment of gastric carcinoma. Ann R Coll Surg Engl 1995; 77:431-436.

4. Miwa K, Miyazaki I, Sahara H, Fujimura T, Yonemura $\mathrm{Y}$ et al. Rationale for extensive lymphadenectomy in 
early gastric carcinoma. Semin Oncl 1996; 23:360-368.

5. Japanese Research Society for Gastric Cancer. Japanese Classification of Gastric Carcinoma, 12th Edition, ed. Nishi M, Omori Y and Miwa K, Kanehara, Tokyo, 1995.

6. Namieno T, Koito K, Higashi T, Sato N, and Uchino J. General pattern of lymph node metastasis in early gastric carcinoma. World J Surg 1996; 20:996-1000.

7. Lee WJ, Lee PH, Yue SC, Chang KC, Wei TC et al. Lymph node metastases in gastric cancer: Significance of positive number. Oncology 1995; 52:45-50.
8. Jaehne J, Meyer HJ, Maschek H, Geerlings H, Burns E et al. Lymphadenectomy in Gastric Carcinoma. A prospective and prognostic study. Arch Surg 1992; 127:290-294.

9. Yamao T, Shirao K, Ono H, Kondo H, Saito D et al. Risk factors for lymph node metastasis from intramucosal gastric carcinoma. Cancer 1996; 77:597-598.

10. Bunt AM, Hogendoorn PC, Van de Velde CJ, Bruijin JA, and Hermans J. Lymph node staging standards in gastric cancers. J Clin Oncl 1995; 13:2309-2316. 